\title{
Combining Low Temperature Fluorescence DNA-Hybridization, Immunostaining, and Super-Resolution Localization Microscopy for Nano-Structure Analysis of ALU Elements and Their Influence on Chromatin Structure
}

\author{
Matthias Krufczik ${ }^{1}$, Aaron Sievers ${ }^{1}$, Annkathrin Hausmann ${ }^{1}$, Jin-Ho Lee ${ }^{1}$, \\ Georg Hildenbrand ${ }^{1,2}$, Wladimir Schaufler ${ }^{3}$ and Michael Hausmann ${ }^{1, *}$ \\ 1 Kirchhoff-Institute for Physics, Heidelberg University, Im Neuenheimer Feld 227, \\ 69120 Heidelberg, Germany; krufczik@kip.uni-heidelberg.de (M.K.); Sievers_Aaron@web.de (A.S.); \\ annkathrin.hausmann@googlemail.com (A.H.); jin-ho.lee@kip.uni-heidelberg.de (J.-H.L.); \\ hilden@kip.uni-heidelberg.de (G.H.) \\ 2 Department of Radiation Oncology, University Medical Center Mannheim, Heidelberg University, \\ Theodor-Kutzer-Ufer 3-5, 68159 Mannheim, Germany \\ 3 German Cancer Research Center (DKFZ), Im Neuenheimer Feld 280, 69120 Heidelberg, Germany; \\ w.schaufler@dkfz.de \\ * Correspondence: hausmann@kip.uni-heidelberg.de; Tel.: +49-6221-54-9824
}

Academic Editor: Herbert Schneckenburger

Received: 16 February 2017; Accepted: 2 May 2017; Published: 7 May 2017

\begin{abstract}
Immunostaining and fluorescence in situ hybridization (FISH) are well established methods for specific labelling of chromatin in the cell nucleus. COMBO-FISH (combinatorial oligonucleotide fluorescence in situ hybridization) is a FISH method using computer designed oligonucleotide probes specifically co-localizing at given target sites. In combination with super resolution microscopy which achieves spatial resolution far beyond the Abbe Limit, it allows new insights into the nano-scaled structure and organization of the chromatin of the nucleus. To avoid nano-structural changes of the chromatin, the COMBO-FISH labelling protocol was optimized omitting heat treatment for denaturation of the target. As an example, this protocol was applied to ALU elements-dispersed short stretches of DNA which appear in different kinds in large numbers in primate genomes. These ALU elements seem to be involved in gene regulation, genomic diversity, disease induction, DNA repair, etc. By computer search, we developed a unique COMBO-FISH probe which specifically binds to ALU consensus elements and combined this DNA-DNA labelling procedure with heterochromatin immunostainings in formaldehyde-fixed cell specimens. By localization microscopy, the chromatin network-like arrangements of ALU oligonucleotide repeats and heterochromatin antibody labelling sites were simultaneously visualized and quantified. This novel approach which simultaneously combines COMBO-FISH and immunostaining was applied to chromatin analysis on the nanoscale after low-linear-energy-transfer (LET) radiation exposure at different doses. Dose-correlated curves were obtained from the amount of ALU representing signals, and the chromatin re-arrangements during DNA repair after irradiation were quantitatively studied on the nano-scale. Beyond applications in radiation research, the labelling strategy of immunostaining and COMBO-FISH with localization microscopy will also offer new potentials for analyses of subcellular elements in combination with other specific chromatin targets.
\end{abstract}

Keywords: combinatorial oligonucleotide FISH (COMBO-FISH); combined immuno-staining; cell nucleus; chromatin arrangement; localization microscopy; ALU-repeats; low LET radiation exposure 


\section{Introduction}

Fluorescence in situ hybridization (FISH) using target-specific DNA probes as well as immunostaining using specific antibodies have each become routine techniques in modern molecular biology, cell research, and medical diagnosis [1]. Experiments combining both techniques simultaneously in combination with high-resolution microscopy (a review about high-resolution microscopy is presented in [2,3]) are still challenging because of the different protocol essentials and their influence on the examined structure [4]. FISH of chromatin usually needs DNA denaturation at high temperatures [5], which should be avoided for analysis of DNA structure with high-resolution microscopy. Mongelard et al. [6] avowed the denaturation step of the target DNA as the most damaging step in the FISH procedures. This destructive effect due to heat treatment was clearly shown by super-resolution scanning near-field optical microscopy (SNOM) [7]. In contrast to DNA-DNA FISH of chromatin in cell nuclei mRNA FISH using appropriate oligonucleotides can be optimally performed at nearly physiological temperatures [8] and applied to super-resolution microscopy $[8,9]$ together with immunostaining. Although the combination of DNA-FISH and immunostaining resulted in apparently well-maintained chromatin morphology [4], it should be considered that the usually preferred conventional preparation techniques in super-resolution microscopy determine the practical limits of application [10]. Fornasiero et al. [11] presented an overview of the advantages and disadvantages of the high-resolution microscopy methods regarding various biological tasks. A challenge that must not be underestimated in this context of circumventing possible limits is the development of adequate computer-based analysis methods for the evaluation of the high-resolution data.

In this work, we present a method to analyze the chromatin organization of ALU elements and its heterochromatic environment in intact cell nuclei using an optimized low-temperature DNA-hybridization-protocol for chromatin FISH in combination with immunostaining, super-resolution localization microscopy, and in-house-developed computer-based methods for structural analysis.

In biomedical research and diagnostics, DNA-FISH is routinely used for the detection of numerical and structural chromosome aberrations like gene copy number changes, translocations, deletions, and amplifications in cytological smear preparations, histological sections, and isolated peripheral blood and bone marrow cells [12]. Usually FISH probes manufactured as BAC or YAC (bacterial/yeast artificial chromosome) probes have a length of some ten up to some hundred kilo bases. For specific targeting, all these established protocols require harsh steps of target denaturation by heat treatment accompanied by chaotropic agents [13,14]. This specimen preparation not only has an influence on the nano-structure of the DNA, but seems to be incompatible with the requirements of immunostaining protocols. In so-called low-temperature FISH experiments, thermal denaturation of the target DNA has been omitted and measurements with scanning near-field optical microscopy (SNOM) have shown persuasive results regarding the preservation of metaphase chromosome morphology by renouncement of denaturation [7]. Based on these low-temperature protocols, a novel technique called combinatorial oligonucleotide (COMBO)-FISH was established and proven by Hausmann et al. [15] (for review, see [16]). Oligonucleotide sets with a length of 15 to 30 bases per probe are used [17] that specifically bind within a designated genomic region. Using home-developed algorithms for genome data bank analysis, a computer search for appropriate unique or different oligonucleotides which co-localize in the desired region(s) [18] can result in a specific label and produce a detectable signal in fluorescence microscopy. Compared to standard FISH probes of several kb of DNA, the small COMBO-FISH probes of about 20 bases only are closely attached to the labelled DNA strand, and the fluorochromes have a very small distance to the target (about $20 \mathrm{~nm}$ linker length). In combination with localization microscopy $[19,20]$, it is possible to get detailed information about the structure of the stained target [21,22].

COMBO-FISH oligonucleotide probes can be developed and synthesized for any given target (e.g., centromeres, genes, breakpoint regions, trinucleotide expansion repeats, etc.), if the species has been sequenced completely for any given organism. Depending on the target and type of 
oligonucleotide, the probe binding can be realized by Watson-Crick or Hoogsteen bonding. The latter requires either pure purine or pure pyrimidine oligonucleotide probes, and results in triple strand binding. DNA denaturation of the target strand by heat treatment can be avoided $[15,23]$. In this case, the structural influence on the chromatin is negligibly low.

An ALU element is a short stretch of DNA (SINE = short interspersed nuclear element) which appears in different kinds and repetition rates in large numbers in primate genomes and other species. Thirty-seven different ALU sequence families are known [24]. They differ in their lengths depending on their evolutionary age, but show a close similarity which can be expressed by the probability of a consensus-sequence in the different families [25]. These ALU elements seem to be involved in gene regulation, genomic diversity [26], disease [24] and DNA repair [27]. Much work on ALUs is focused on the sequence information, but for the understanding of regulatory mechanisms, it may also be necessary to study the organization, structure, and distribution of ALU elements in the nucleus to elucidate their role for chromatin modelling, functionally correlated structure formation, and other effects. Using chromosome conformation capture techniques like Hi-C [28], mega-base-sized chromatin-interaction domains were found and seem to be a structural feature of the genome organization. ALU elements seem to be enriched at boundary regions of chromosome interaction domains, indicating a role in the spatial organization of the genome [29,30]. In addition, ALU elements are embedded in prominent oncogenes (e.g., BRCA1) [31]. During evolution, ALU elements as transposonable elements seem to play a prominent role in genome adaptation to environmental stress [32], resulting in many different functions currently known [33]. The "network-like" organization of ALU-elements over the whole genome makes them an ideal candidate for the description of genome-wide topological changes induced by environmental pressure such as radiation treatment.

Localization microscopy has been realized in several different forms [3], such as photoactivated localization microscopy (PALM) [34], fluorescence photoactivation localization microscopy (F)PALM [35], stochastic optical reconstruction microscopy (STORM) [36,37], direct stochastic optical reconstruction microscopy (dSTORM) [38], ground state depletion microscopy followed by individual molecule return (GSDIM) [39], or super-resolution optical fluctuation imaging (SOFI) [40], etc. As a technique of single molecule imaging, it gets full benefit from COMBO-FISH, which can be used for nano-probing with single dye molecules [21,22]. Implementation of COMBO-FISH probes into a global cell nucleus network like heterochromatin can be applied to get additional structural information about certain regions of the nucleus in the frame of the chromatin network. Localization microscopy uses optical isolation of the fluorophores to circumvent the Abbe diffraction limit of optical resolution. Differently colored or switchable fluorophores can be used to achieve optical isolation. SPDM ("spectral position determination microscopy") [19]—one type of localization microscopy—uses a property of many fluorophores [41]: During illumination with high power lasers $\left(\mathrm{kW} / \mathrm{cm}^{2}\right)$, these fluorophores are reversibly bleached. With a certain probability, they recover from the bleached state to become fluorescent again. The stochastic recovery of fluorophores from the dark state yields to an optical isolation by "blinking", which allows a precise position determination far below the Abbe limit. Acquisition of a high number of images over time results in a good reconstruction of the original distribution of fluorescent markers [42].

The raw data for a localization image consist of a large number of images in an image stack acquired in a time series. According to the mentioned principle of localization microscopy, on every image of the image stack, only a few fluorophores are fluorescently switched on. This is used to compute the exact positions of the fluorophore. A point signal is imaged by a microscope into an Airy disc according to the point-spread function (PSF). In practice, the intensity of the maximum of this PSF must be at least four times higher than the background intensity to get registered as an event. The area of increased intensity must fit into a square region of interest (ROI) because the PSF has a round shape. The double of the background intensity is subtracted from each pixel of the ROI. The resulting reduced ROI is effectively rounded. The center of gravity $\mu$ and the associated standard deviation $\sigma$ of the signal are determined using two-dimensional Gaussian function $f$. From this, the localization precision 
$\Delta \mu$ can be calculated, which depends among others things (compare formulas below), on the number of detected photons $q_{i}$, and the intensity background $N_{B}$. A more detailed description can be found in [43].

$$
\begin{gathered}
f(\vec{x})=\frac{Q}{2 \pi \sigma_{x} \sigma_{y}} e^{-\frac{\left(\frac{x-\mu_{x}}{\sigma_{x}}\right)^{2}+\left(\frac{y-\mu_{y}}{\sigma_{y}}\right)^{2}}{2}} \\
Q=\sum q_{i} \quad \vec{\mu}=\sum \frac{q_{i}}{Q} \vec{x}_{i} \quad \sigma_{x}^{2}=\sum\left(x_{i}-\mu_{x}\right)^{2} \frac{q_{i}}{Q} \\
\Delta \mu_{x}=\sqrt{\frac{1}{12 Q}+\sum\left(\frac{x_{i}-\mu_{x}}{Q}\right)^{2}\left(q_{i}+N_{B}\right)}
\end{gathered}
$$

This results in a matrix which contains the coordinates for every measured fluorophore with a typical localization precision of about $10 \mathrm{~nm}$ [44]. The pointillist localization image can be used for further analyses based on distance and frequency measurements (e.g., [45,46]). Usually, density images (see Figure 1 for the different possible methods to produce images from the localization data) are used. The images represent a form of visualization, and due to clarity of structure recognition, do not always require highest resolution. Nevertheless, the full resolution is used for further quantification of nano-structure analyses like cluster formation, etc. [46,47].

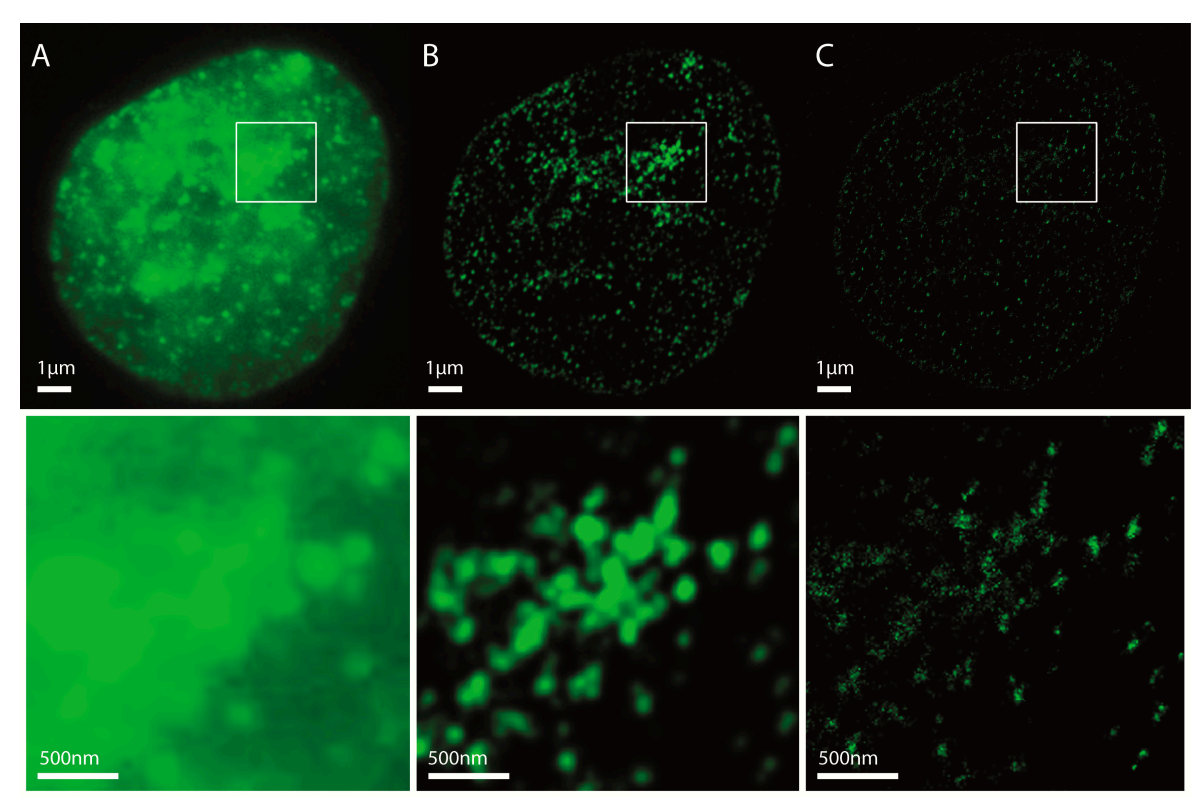

Figure 1. Localization microscopy images of $\mathrm{H} 3 \mathrm{~K} 9 \mathrm{me} 3$ immunostaining in a $\mathrm{SkBr} 3$ cell nucleus. (A) Conventional widefield microscopy image; (B) Density image. The image is created from the coordinate matrix. For this purpose, the localization data stored in nanometres in the coordinate matrix must be converted into pixels. For this, a pixel size in nm must be determined. The coordinates from the matrix are then assigned to the corresponding pixels. Then, in a fixed radius $R$ around each coordinate, it is determined how many further coordinates are within this radius. This value is assigned to the coordinate. In order to emphasize contiguous structures, each coordinate with an assigned value greater than zero is the starting point of a Gaussian distribution with a given $\sigma$. The sum of all Gaussian distributions then represents the intensity distribution of the Gaussian-filtered density image (example: Pixel size $=10 \mathrm{~nm} /$ pixel, radius $R=1000 \mathrm{~nm}$, Gaussian filter $\sigma=50 \mathrm{~nm}$ ); (C) Standard localization image. This image is also created from the localization data. Again, a pixel size must be determined. Here, every coordinate is the starting point of one Gaussian distribution with the localization precision as $\sigma$. (pixel size $=10 \mathrm{~nm} /$ pixel). 


\section{Results}

\subsection{Localization Microscopy after Immunohistochemistry Labelling}

Localization microscopy data result in a matrix of coordinates of the detected signals. This list also contains a set of other data, such as intensity, localization precision, errors of the values, etc. By means of pointillist visualization (for details of representation, see Figure 1) of the detected labelling molecule loci, localization microscopy offers an optical image scaled by the applied pixel size. Compared to imaging using the same setup in a conventional wide-field mode, the resolution is much better. Better resolution means that the minimal detectable point distance is considerably smaller as compared to conventional optical far-field microscopy. Localization microscopy can optically distinguish fluorophores with a minimum distance on the order of $10 \mathrm{~nm}$. On the other hand, conventional microscopy requires a minimum distance of about $200 \mathrm{~nm}$ for the separation of two points. Figure 1 shows localization images of $\mathrm{H} 3 \mathrm{~K} 9 \mathrm{me} 3$ immunostaining in comparison to conventional microscopy. Here, H3K9me3 was used as a heterochromatin marker [48], especially due to its staining efficiency.

\subsection{Probe Design for ALU-Combinatorial Oligonucleotide (COMBO)-Fluorescence In Situ Hybridization (FISH)-Staining}

For the sequence of the ALU COMBO-FISH oligonucleotide probe used, genome data base studies were performed. The typical ALU consensus sequence is about 300 bp long (5'-3') [25]: GGCCGGGCGCGGTGGCTCACGCCTGTAATCCCAGCACTTTGGGAGGCCGAGGCGGGCGGATC ACCTGAGGTCAGGAGTTCGAGACCAGCCTGGCCAACATGGTGAAACCCCGTCTCTACTAAAAA TACAAAAATTAGCCGGGGCGTGGTGGCGCGCGCCTGTAATCCCAGCTACTCGGGAGGCTGAG GCAGGAGAATCGCTTGAACCCGGGAGGCGGAGGTTGCAGTGAGCCGAGATCGCGCCACTGC ACTCCAGCCTGGGCGACAGAGCGAGACTCCGTCTC.

COMBO-FISH probes are usually about $20 \mathrm{bp}$ long, and must fit perfectly to the target region. For that reason, it was necessary to choose a part of the ALU consensus sequence which has as many targets in the genome as possible, but only in the ALU regions. With the help of special in-house software, a 17 bp probe was found (5'-3') (TAATCCCAGCACTTTGG) binding to the complementary strand by Watson-Crick bonding, since it consists of purine and pyrimidine nucleotides. It was checked against the whole genome to determine whether the probe only binds in ALU regions. A map was computed which schematically shows this expected distribution of this ALU probe along the human chromosomes (Figure 2). The selected ALU probe sequence appears 385,924 times in the genome. The number of hits of the consensus sequence was 1,180,685. Of all the 17mer probe sequences, $99.9 \%$ are located within consensus sequences. Thus, the probe covers approximately $30 \%$ of the ALU elements - a proportion that is sufficient to represent the distribution of the ALU elements microscopically.

For comparison, another 17mer was arbitrarily selected from the L1-elements (GGTGATTTCTGCATTTC) and depicted in the same way (Figure 2). L1 belongs to the LINEs (long interspersed nuclear elements). The frequency density shows a completely different distribution, indicating that the frequency distributions are specific for any given 17mer sequence.

The oligonucleotide COMBO-FISH ALU probe carrying one fluorochrome (Alexa 568) at the $5^{\prime}$ end was manufactured commercially and hybridized according to the protocol described below. As a control, we also designed the most frequently occurring unique COMBO-FISH probe of the L1-elements according to the same procedure as for ALU sequences, and applied this probe together with the ALU COMBO-FISH probe to cell nuclei. The probe tags of ALU and L1 elements did not co-localize in the localization image (data not shown and will be published in detail elsewhere).

The successfully designed uniquely-binding COMBO-FISH ALU probe was applied on the breast cancer cell line SkBr3 cells. On widefield images, by visual inspection, the fluorescence results are comparable to other ALU-studies prepared by conventional microscopy [49]. 


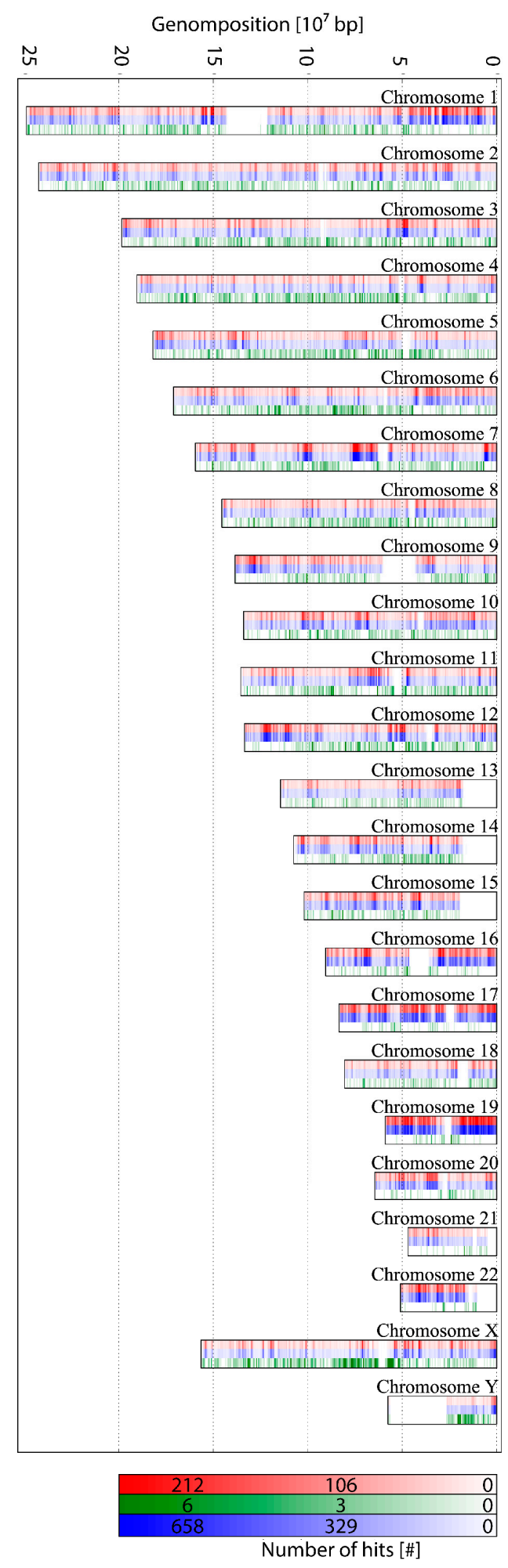

Figure 2. ALU-distribution along the genome: The intensity of the bars indicates the frequency within a $500 \mathrm{~kb}$ section of the given chromosome. Red: Position of the designed 17mer ALU probe. The sequence associated with the ALU probe appears in the entire genome at different frequency densities. Blue: Corresponding positions of the ALU consensus sequence. The number of emergence of the $17 \mathrm{mer}$ probe sequence was compared with the density of the ALU consensus sequence by using the program Repeatmasker [50]. Green: Arbitrarily selected 17mer from the L1 element. Although this 17mer appears very often in the genome in contrast to any randomly-chosen $17 \mathrm{mer}$, the frequency density is significantly different from the selected ALU consensus 17mer. 


\subsection{Two-Color Localization Microscopy and ALU Clustering}

Two-color localization microscopy was applied to further analyze the quality of the protocol and to simultaneously quantify the nano-structural arrangements of ALU repeats and heterochromatin (Figure 4). First, it was shown that the amount of co-localization between ALU and heterochromatin is negligibly low, as expected [51]. Furthermore, it was proofed that ALU sequence-dense regions are spatially associated but preclusive to labelled heterochromatin (Figure 4) [52].

Cluster analysis software was applied to the localization images [46,47]. The algorithm identifies "cluster-points" within the localization data according to user-defined parameters. These parameters are the minimum number of fluorescence signals within a defined circular region around each blinking event and the radius of this region. An event is identified as a "cluster-point" if at least as many points as defined as minimum points are in the predefined radius. The remaining points are identified as "noise-points". The points are then divided into clusters. If two cluster-points have a smaller distance than the given radius, they belong to one cluster. In addition, all noise-points whose distance to a cluster-point is smaller than the radius also belong to the cluster. This cluster search algorithm is called density-based spatial clustering of applications with noise (DBSCAN) [53]. In this case, this allows the identification of ALU sequence-dense regions.

Here, a radius of $100 \mathrm{~nm}$ and minimum point number of 10 was used to identify "ALU cluster", which means that there is a compact area of many repetitive ALU elements. After cluster recognition, the centers of the ALU clusters were computed with the Surveyor's Area Formula [54]. These centers were used as the center points of increasing circle shells with the size of $10 \mathrm{~nm}$. The heterochromatin density in these shells was computed (Figure 3) (heterochromatin density = number of heterochromatin points/area of the corresponding circle shell).

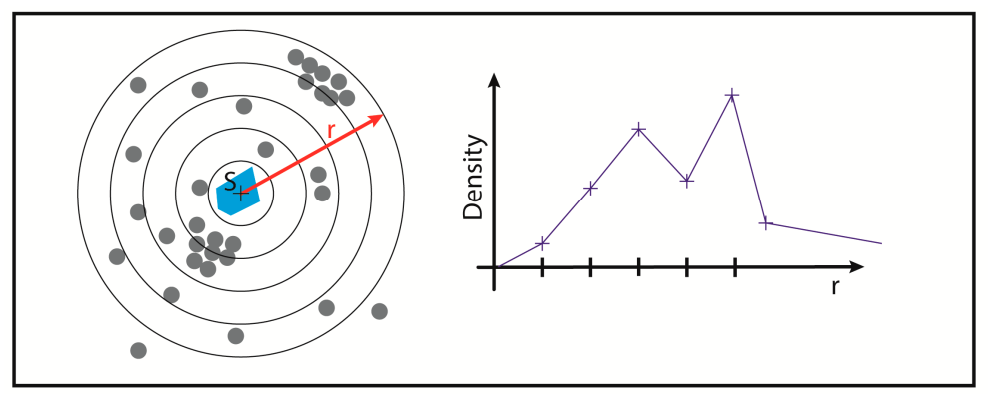

Figure 3. Density determination around ALU clusters. A cluster identified by density-based spatial clustering of applications with noise (DBSCAN) is shown in blue. Around the center of gravity S of this cluster, the density (in number of points per area) is now determined in concentric circular shells with a fixed thickness.

The results show that ALU and heterochromatin are spatially associated but do not co-localize or intermingle (Figures 4-6). Higher densities of heterochromatin are found within the near neighborhood of ALU clusters, but almost never in a co-localization, which indicates an intermingling arrangement. This corresponds to the results from other studies [49,52,55]. As an example, a typical density distribution for a cell nucleus is shown in Figure 5. 


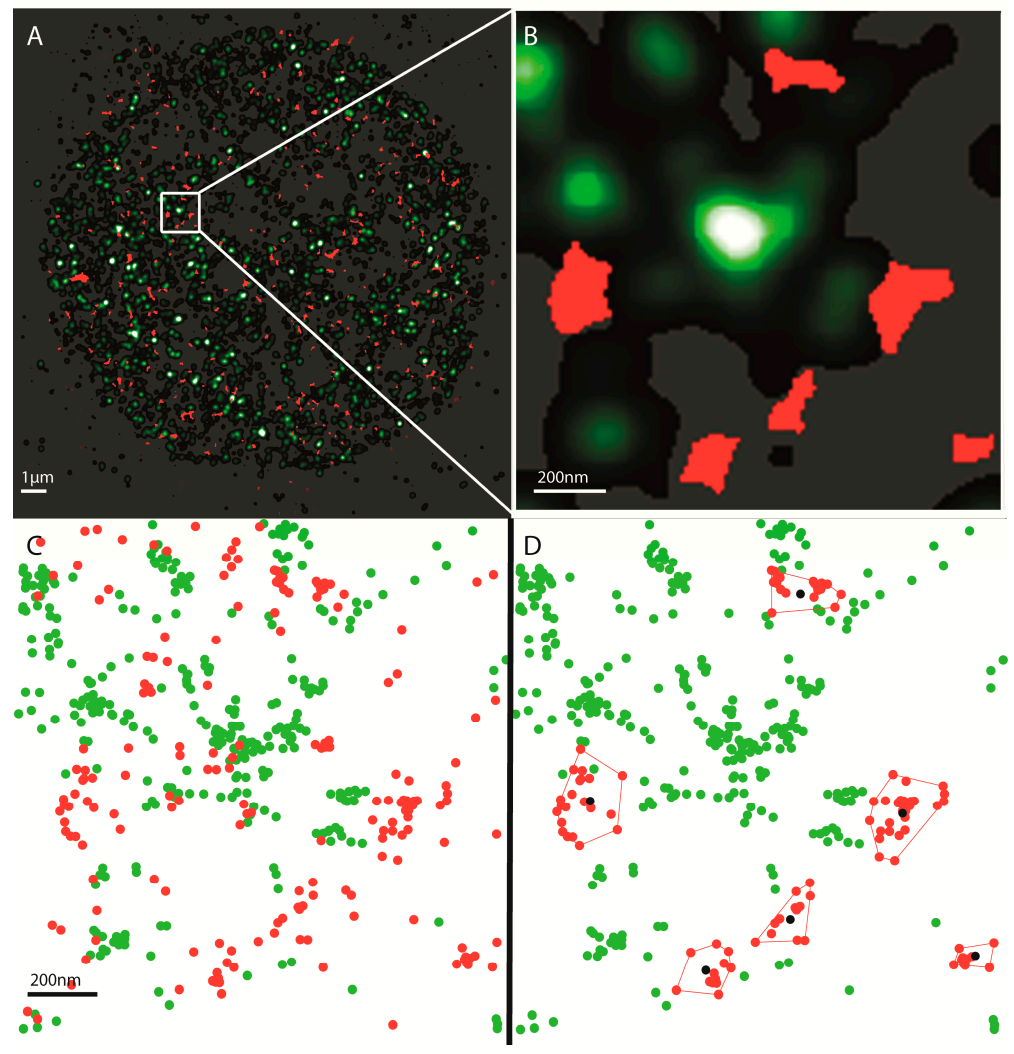

Figure 4. Image of the ALU clusters labelled by combinatorial oligonucleotide fluorescence in situ hybridization (COMBO-FISH) probes (red) and heterochromatin labelled by H3K9me3 antibodies (green). (A) Density image: Pixelsize $=10 \mathrm{~nm} /$ pixel, radius $=1000 \mathrm{~nm}$, Gaussian filter $\sigma=50 \mathrm{~nm}$; (B) Zoom of the marked region indicating the closed point patterns; (C) and (D) binary depiction of the same region shown in (B); (C) Every point corresponds to a single fluorophore; (D) ALU clusters and their barycentre (black) are shown.

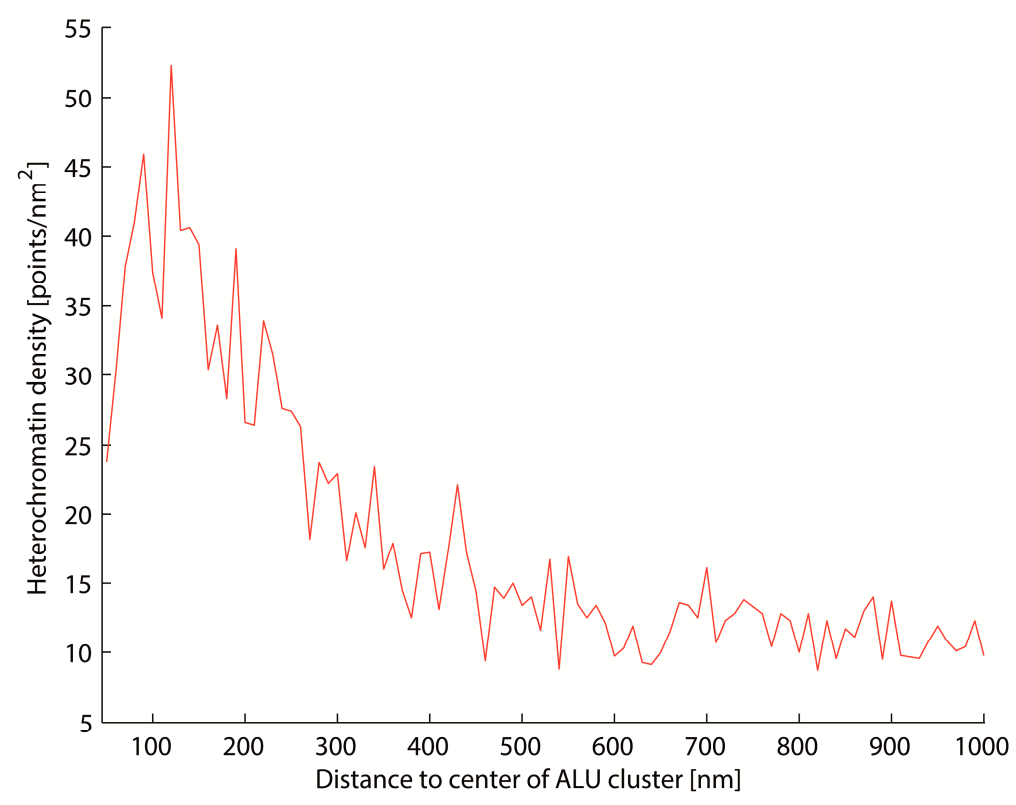

Figure 5. Mean heterochromatin density around ALU clusters for one cell. Approximately 200 ALU clusters were detected per cell. The density distribution for every ALU cluster was determined, and the mean value was computed. 


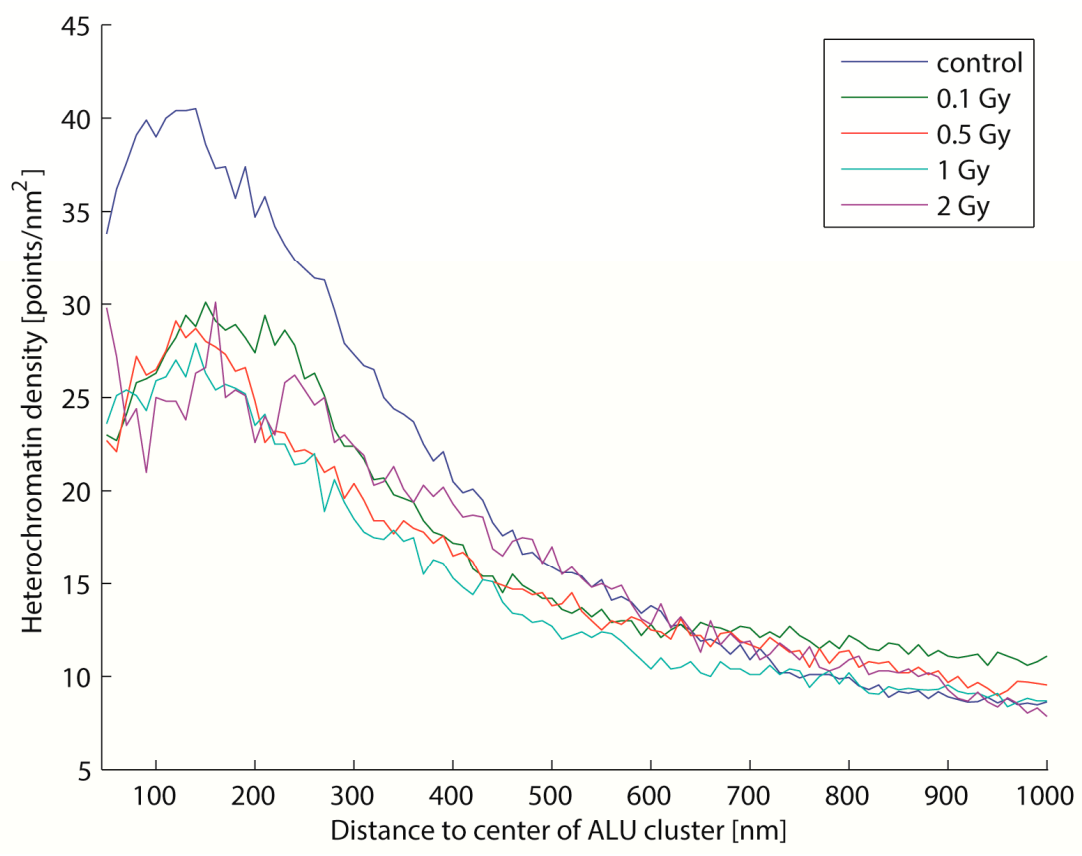

Figure 6. Mean heterochromatin density around ALU clusters after irradiation with different doses (about 200 ALU clusters were detected per cell, 30 to 40 cells were analysed per dose value). The density distribution for every ALU cluster was determined, and the mean values were computed. The data shows that irradiation influences the heterochromatin density around ALU clusters. A clear difference between irradiated and non-irradiated cells is visible, indicating a de-compaction. However, the underlying mechanism seems to be independent of the dose, since no considerable differences are visible between the irradiations ( 0.1 to $2 \mathrm{~Gy}$ ).

\subsection{Biological Dosimetry by ALU Point Counting}

The combination of COMBO-FISH and localization microscopy allows the detailed analysis of the number of ALU elements in the nucleus. At a first glimpse, the measured number of points does not equal the real number of ALU consensus elements if one estimates the amount of points that could occur in the whole 3D nucleus from Figure 4. Hybridization and measurement efficiency reduce the amount of detected ALU oligonucleotide labels. Assuming a constant hybridization and measurement efficiency for all experiments, the absolute numbers detected and its dose-dependent decrease could be estimated to be proportional to the real values. This offers an opportunity for biological dosimetry (Figure 7). The number of ALU COMBO-FISH signals detected in a nucleus inversely follows the dose increase. By appropriate fitting, we were able to prepare a dose-effect curve with the following parameters:

$$
N(D)=\mathrm{a} D^{2}+\mathrm{b} D+\mathrm{c}
$$

where $N$ means the number of detected points (= ALU sequences), $D$ the radiation dose, and $\mathrm{a}, \mathrm{b}, \mathrm{c}$ empirical constants of the fit (see the figure legend). The data show that irradiation may functionally or structurally (maybe by dimerization) influence the ALU sequences so that the accessibility of the probes is reduced.

Irradiation also changes the density distribution of heterochromatin around ALU clusters (Figure 6). The difference between the irradiated cells and the control cells is remarkable. It seems that irradiation changed the conformation of the chromatin structure and led to a decrease of heterochromatin density (de-compaction of the labelled sites) in the near-environment around the ALU cluster. This is in good agreement with recently published data $[45,56]$. 


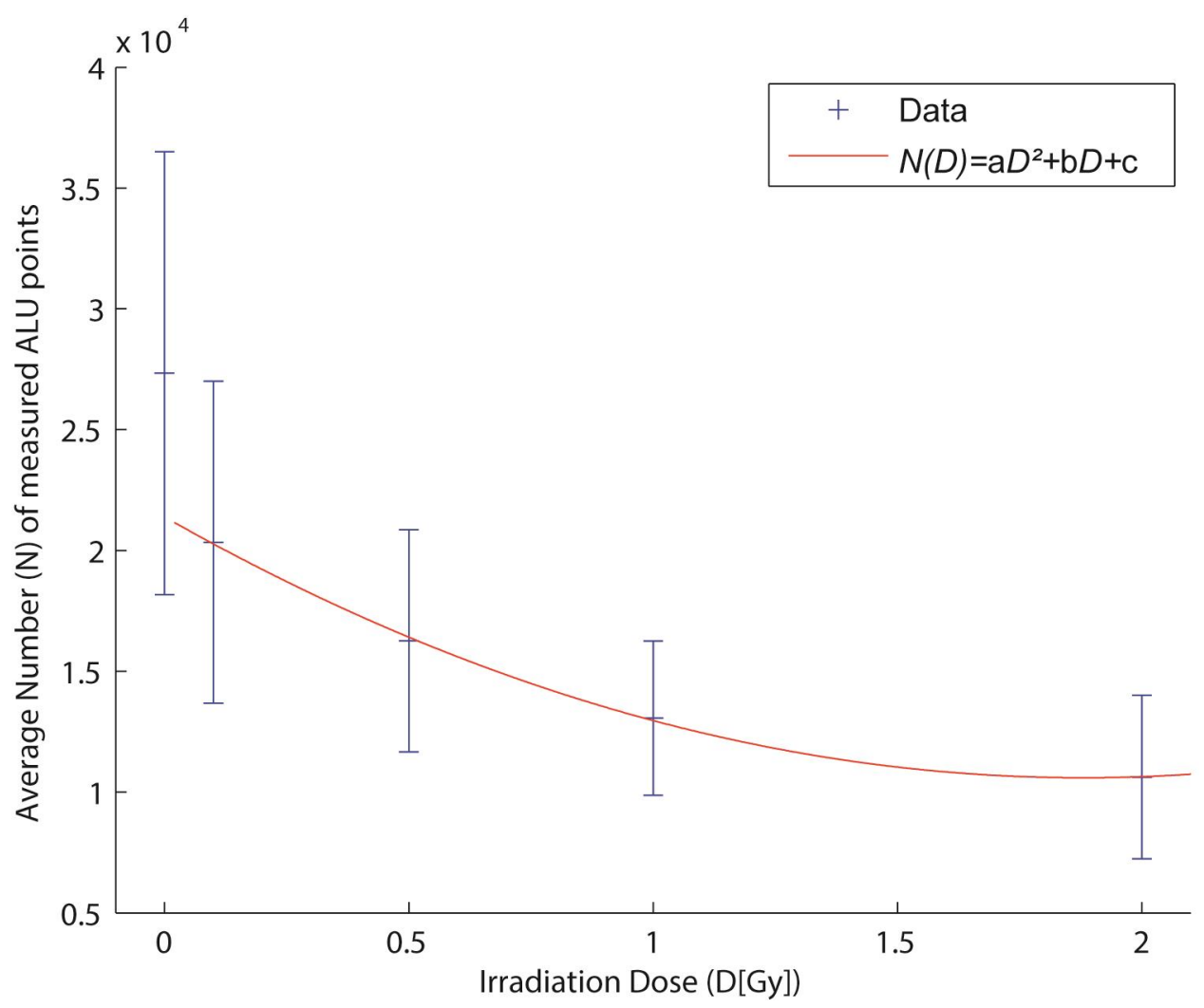

Figure 7. Average number of measured ALU elements relative to the radiation exposure dose as detected by specimen fixation 30 min after irradiation with $6 \mathrm{MeV}$ photons. The measurement data is fitted by a linear-quadratic curve $\left(N(D)=a D^{2}+b D+c\right)$ with the parameters $\mathrm{a}=3054, \mathrm{~b}=-1.148 \times 10^{4}$, $c=2.139 \times 10^{4}$.

\section{Discussion}

Usually, COMBO-FISH is used to specifically stain small units of the genome (e.g., a given centromere or a given gene of a chromosome) [21-23,57]. In this work, we used a uniquely specific COMBO-FISH probe to stain dispersed ALU consensus regions which can be found distributed all over the whole genome. By means of this probe, the distribution of ALU shows a kind of a "network", as depicted by the pointillist visualization of localization microscopy. By recognizing ALU sequence-dense regions using a cluster search algorithm and analyzing the heterochromatin density, a characteristic preclusive association between ALU and heterochromatin was shown. It remains a challenge to find methods to analyze and mathematically quantify structures such as these and their changes. This would be necessary for analyses of effects on chromatin re-organization during and after environmental treatment such as irradiation. Novel theoretical procedures will be tasks of future projects. In this article, we have shown that the combination of labelling methods based on our newly-adapted protocols and super-resolution microscopy allows appropriate imaging for the quantification of structural parameters, and offers a variety of novel applications. Unfortunately, routine applications are mostly done on two dimensional (2D) optical sections, since 3D localization microscopy is still a challenge - especially for labelling stability and detection speed. Since the 2D data obtained represent a projection of a layer approximately $500 \mathrm{~nm}$ thick, they can be assumed to contain in part 3D information.

In this work, we were able to apply uniquely-binding purine-pyrimidine COMBO-FISH probes without heat treatment for specific chromatin labelling in cell nuclei, and to combine this technique with simultaneous immunostaining in the nuclei. Since this probe does not bind as a triple strand, not all structure-influencing steps could be completely dispensed (e.g., chemical denaturation with 
hydrochloric acid). Omitting heat denaturation avoids the most chromatin-destructive step, and represents a considerable improvement [6,7]. In principle, the COMBO-FISH protocol presented here might also be applied to standard FISH probes (e.g., BAC-probes) and thus combined with immunohistochemical staining. However, commercially-available FISH-probes are delivered in a ready-to-use buffer mix that is adapted to protocols including heat denaturation [5]. Moreover, the longer such a BAC-probe is, the more it requires a denaturation of a longer part of the DNA strand, which only seems to possible by heat or harsh chemical treatment [58].

Here, the stained cells were not exposed to such harsh structure-changing steps in comparison to standard methods using commercially-available probes. It is possible to get the full benefit of the improved resolution of localization microscopy with these methods, since nano-scaled structuring is mainly maintained in the native form. These localization data were evaluated using specially optimized analytical computer algorithms.

The mentioned results show a spatial arrangement of ALU consensus sequences and heterochromatin which has been so far impossible to investigate on the single cell level without high-resolution microscopy. At this point, we were able to microscopically confirm the hypothesis that heterochromatin and ALU do not co-localize, but may associate functionally in a certain way. We also gained some information about the structural connection of ALU and heterochromatin, which could contribute to a better understanding of chromatin interaction mechanisms after irradiation and during repair $[52,59,60]$. Since our major goal was methodological development, we used the SkBr3 cell line as an easy-to-handle but well-established cell line in breast cancer research [61]. Nevertheless, the conclusions drawn-especially by the application of the H3K9me3 antibody—should be verified by other cell lines (i.e., ideally, normal cells). This, as well as the study of our approach of biological dosimetry in different cell systems will be tasks of future investigations.

Although the dose-correlated density measurements have only been shown for one cell type, the reproducible sensitivity of the applied quantification indicates the potential of sensitive biological dosimetry, and therefore offers a broad spectrum of future investigations. However, further research seems to be necessary to understand the mechanisms behind the inverse correlation of ALU signals and dose. Whether there may be a pure methodological reason due to probe accessibility or a more complex reason based on biological mechanisms of chromatin modification and association, the reproducible dose-effect correlation offers alternative approaches to biological dosimetry beyond metaphase or micro-nuclei analyses.

\section{Materials and Methods}

In the following, the developed protocol for the combination of COMBO-FISH and immunostaining is described:

\subsection{Staining Materials and Concentrations (Immunostaining and COMBO-FISH)}

$0.1 \mathrm{M} \mathrm{HCl}$ in $\mathrm{H}_{2} \mathrm{O} ; 1 \times$ Phosphate-buffered saline buffer (PBS) $+\mathrm{MgCl}_{2}(0.901 \mathrm{mM}) / \mathrm{CaCl}_{2}$ $(0.493 \mathrm{mM}) ; 0.05 \%$ Triton-X in $1 \times$ PBS $+\mathrm{Mg} / \mathrm{Ca} ; 2 \times$ Saline-sodium citrate buffer (SSC) $(\mathrm{pH} 7.4) ; 4 \%$ Formaldehyde solution (in $1 \times \mathrm{PBS}+\mathrm{Mg} / \mathrm{Ca}$ ); $2 \%$ Formaldehyde solution (in $1 \times \mathrm{PBS}+\mathrm{Mg} / \mathrm{Ca}$ ); Blocking solution $(2 \%$ BSA in $1 \times \mathrm{PBS}+\mathrm{Mg} / \mathrm{Ca})$; Permeabilisation solution $(0.2 \%$ Triton- $\mathrm{X}$ in $1 \times \mathrm{PBS}+\mathrm{Mg} / \mathrm{Ca}$ ); 4',6-Diamidino-2-phenylindole (DAPI)-solution (100-500 ng/mL in $1 \times \mathrm{PBS}) ; 50 \%$ Formamide in $2 \times$ SSC; Object slides; Cover slips (1,5\#) $24 \times 24$ mm; Fixogum; Embedding medium ProlongGold (ThermoFischer, Massachusetts, USA, ProLong ${ }^{\circledR}$ Gold Antifade Mountant, P36930); COMBO-FISH probe solution (IBA life science, Goettingen, Germany, ALU sequence: Alexa Fluor ${ }^{\circledR}$ 568-TAATCCCAGCACTTTGG; L1 sequence: Alexa Fluor ${ }^{\circledR}$ 488-GAAATGCAAATCAAAAC): 200 ng probes in $20 \mu \mathrm{L}$ Tris- $\mathrm{HCl}$ ( $\mathrm{pH}$ 7.4); Primary Antibody: H3K9me3 (concentration: $1.4 \mathrm{mg} / \mathrm{L}, \mathrm{Abcam}$ plc, Cambridge, UK, Anti-Histone H3 (tri methyl K9) antibody-ChIP Grade (ab8898)); Secondary Antibody: A488 (concentration: $4 \mathrm{mg} / \mathrm{L}, \mathrm{Abcam}$ plc, Goat Anti-Rabbit IgG H\&L (Alexa Fluor ${ }^{\circledR} 488$ ) (ab150077)); Humid heat chamber $\left(37^{\circ} \mathrm{C}\right)$; Shaker. 


\subsection{Handling Steps}

The cells were grown on coverslips to about $80 \%$ confluency in the appropriate medium required for the cell type or cell line to be investigated. The cells were washed in $1 \times \mathrm{PBS}+\mathrm{Mg} / \mathrm{Ca}$ for $5 \mathrm{~min}$. After that, the cell specimen was fixed in $4 \%$ formaldehyde (in $1 \times \mathrm{PBS}+\mathrm{Mg} / \mathrm{Ca}$ ) for $10 \mathrm{~min}$ at $37^{\circ} \mathrm{C}$. After washing three times in $1 \times \mathrm{PBS}+\mathrm{Mg} / \mathrm{Ca}$ for $5 \mathrm{~min}$ on a shaker, the cells were incubated in permeabilisation solution for $3 \mathrm{~min}$ on a shaker followed by additional washing three times in $1 \times \mathrm{PBS}+\mathrm{Mg} / \mathrm{Ca}$ on a shaker for $5 \mathrm{~min}$ and incubation into the blocking solution for $30 \mathrm{~min}$. Incubation with the primary antibody in a humidified chamber at $37^{\circ} \mathrm{C}$ for $30 \mathrm{~min}$ and washing three times in $1 \times \mathrm{PBS}+\mathrm{Mg} / \mathrm{Ca}$ on a shaker for $5 \mathrm{~min}$ was then followed by incubation with the secondary antibody in a humidified chamber at $37^{\circ} \mathrm{C}$ for $30 \mathrm{~min}$ and washing three times in $1 \times \mathrm{PBS}+\mathrm{Mg} / \mathrm{Ca}$ on a shaker for $5 \mathrm{~min}$. The specimen was again fixed in $2 \%$ formaldehyde solution (in $1 \times \mathrm{PBS}+\mathrm{Mg} / \mathrm{Ca}$ ) at $37^{\circ} \mathrm{C}$ for $10 \mathrm{~min}$ and washed three times in $1 \times \mathrm{PBS}+\mathrm{Mg} / \mathrm{Ca}$ on a shaker for $5 \mathrm{~min}$. The cells were incubated in $0.1 \mathrm{M} \mathrm{HCl}$ for $10 \mathrm{~min}$ and washed three times in $0.05 \%$ Triton- $\mathrm{X}$ in $1 \times \mathrm{PBS}+\mathrm{Mg} / \mathrm{Ca}$ on a shaker for $5 \mathrm{~min}$. After two steps of incubation in $2 \times$ SSC ( $5 \mathrm{~min}$ ) and in $50 \%$ formamide in $2 \times$ SSC (30 min), $20 \mu \mathrm{L}$ of COMBO-FISH probe solution was given on a slide. The oligonucleotides in the probe solution have to be determined by computer search and synthesized with high purity (for details of computer search, see above). The coverslips with cells were put on the slide and sealed with rubber cement (Fixogum) for incubation in a humidified chamber at $37^{\circ} \mathrm{C}$ for $24 \mathrm{~h}$. After removing the Fixogum and washing three times in $2 \times \mathrm{SSC}$ at $37^{\circ} \mathrm{C}$ on a shaker for $5 \mathrm{~min}$, the specimen was incubated in $1 \times \mathrm{PBS}+\mathrm{Mg} / \mathrm{Ca}$ for $5 \mathrm{~min}$, and the chromatin was counterstained with DAPI for $5 \mathrm{~min}$. Finally, the specimen was washed twice in $1 \times \mathrm{PBS}+\mathrm{Mg} / \mathrm{Ca}$ on a shaker for $5 \mathrm{~min}$ and embedded in $15 \mu \mathrm{L}$ ProlongGold (ThermoFischer) embedding medium on a new slide. After sealing with nail polish, the specimen can be stored at $4{ }^{\circ} \mathrm{C}$ and should be used for localization microscopy within the next fortnight.

\subsection{Microscopy Setup}

The microscope (Figure 8) was built at the Light Microscopy Facility of the German Cancer Research Center in Heidelberg. Four lasers with wavelengths of 405, 491, 561, and $642 \mathrm{~nm}$ are available. The mirror S1 and the dichroic mirrors D1, D2, and D3 bring the four lasers to a common optical axis. An acousto-optic tunable filter (AOTF) is used to select the laser wavelength and set the intensity. Since-in contrast to adjustable filters-no mechanical movement is required, the beam path is stable when changing the wavelength and changing the intensity. In the case of measurements of the same sample with different lasers, this reduces the displacements between the images. Lenses L1, L2, and L3 expand the beam since the subsequent lens system for homogenizing the beam intensity (consisting of lenses L4, L5, L6, and L7) requires a laser beam of fixed diameter. Homogenization is important for localization microscopy because the reversible photobleaching is dependent on the laser intensity, and therefore a homogeneous illumination is assumed. Various mirrors (S) serve to change the direction of the beam. Through the focusing lens, L8, and the mirror, S5, the beam is directed into the objective by the dichroic mirror, D4. A $100 \times$ oil immersion lens with a numerical aperture of NA $=1.46$ is used. The light captured by the lens is imaged onto the EmCCD-camera by the dichroic mirror, D4, a tube lens, TL, a blocking filter, F1, the lens system (L9 and L10 or L9 and L11), and the dichroic filter, D5. The system used here did not yet have the tube lens, the second camera, or the dichroic mirror D5. Here the laser with the wavelength $491 \mathrm{~nm}$ and $561 \mathrm{~nm}$ were used. In localization mode, the laser intensity was $3 \mathrm{KW} / \mathrm{cm}^{2}(491 \mathrm{~nm})$ and $5 \mathrm{~kW} / \mathrm{cm}^{2}(561 \mathrm{~nm})$. The exposure time was $100 \mathrm{~ms}$ per frame. Two thousand frames were captured in each channel. 


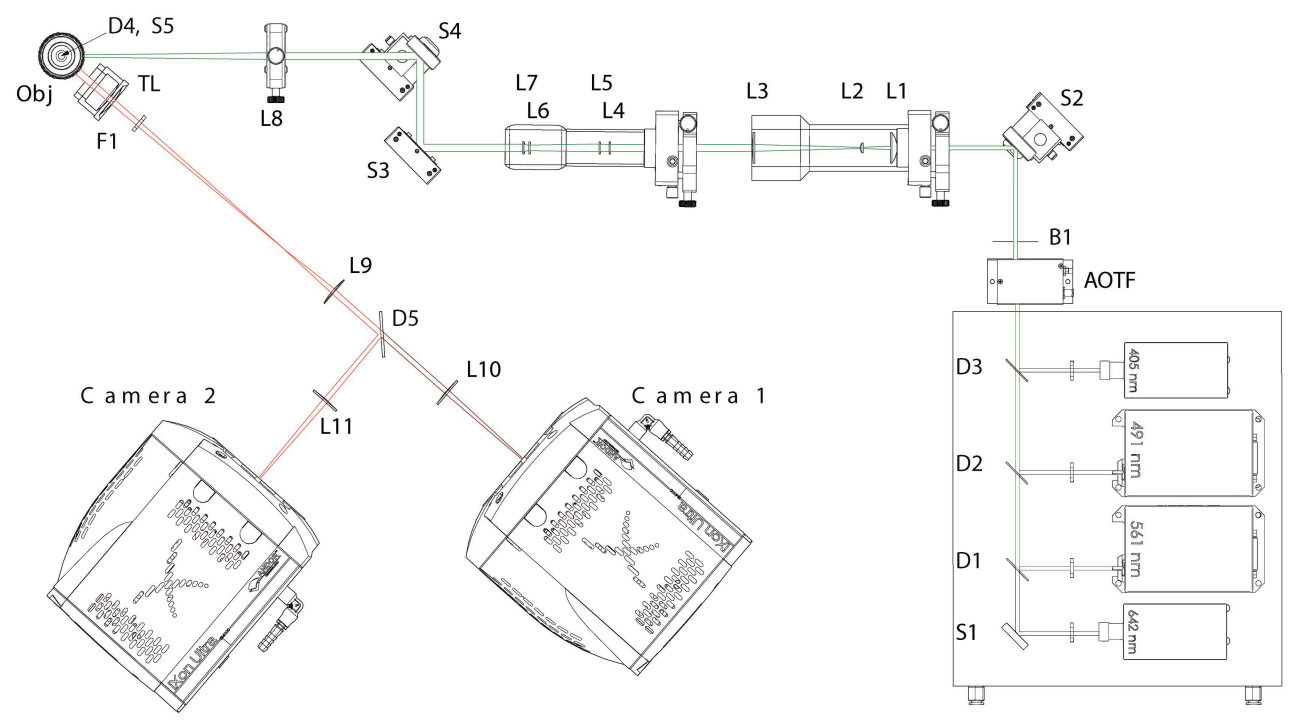

Figure 8. Schematic representation of the microscopic setup (see text for details).

\section{Conclusions}

The application of localization microscopy offers new insights into chromatin conformation on the nanoscale. Quantitative analyses of chromatin conformation changes also require appropriate techniques of nano-probing since voluminous probes might impact the native conformational organization. In order to combine different nano-probing techniques like antibody-labelling or oligonucleotide sequence labelling we developed a low temperature protocol that allows COMBO-FISH labelling and immune-staining simultaneously. Based on this development we were able to show dose dependent conformation changes of ALU sequence clusters and heterochromatin. The results show the new approach towards biological dosimetry using conformational changes as dose related parameter. In contrast to well established techniques of biological dosimetry, this approach, although requiring super-resolution microscopy has the advantage that the number of cells necessary for analyses can be reduced.

Acknowledgments: The financial support by Deutsche Forschungsgemeinschaft and Ruprecht-Karls-Universität Heidelberg within the funding program Open Access Publishing is gratefully acknowledged. The authors acknowledge the constant encouragement by Magnus Foerßer-Mohr and Ina Dischen, both IRRE, Institute of Research Rating and Enhancement, Altenburschla, Germany, to enrich our manuscript by further aspects.

Author Contributions: Matthias Krufczik developed the protocol, processed, refined data, developed MATLAB-scripts and wrote the manuscript. Matthias Krufczik, Annkathrin Hausmann, Jin-Ho Lee did the specimen preparation and the microscopy work. Aaron Sievers did the genome sequence information studies and designed the probe sequences. Wladimir Schaufler built up the localization microscope. Georg Hildenbrand was the scientific advisor and was involved in study design. Michael Hausmann designed the study, was the scientific supervisor and the coordinating investigator. All authors discussed the results and commented on the manuscript.

Conflicts of Interest: The authors declare no conflict of interest.

\section{References}

1. Hausmann, M.; Cremer, C. Standardisation of FISH-procedures: Summary of the first discussion workshop. Anal. Cell. Pathol. 2003, 25, 201-205. [CrossRef] [PubMed]

2. Schermelleh, L.; Heintzmann, R.; Leonhardt, H. A guide to super-resolution fluorescence microscopy. J. Cell Biol. 2010, 190, 165-175. [CrossRef] [PubMed]

3. Cremer, C.; Masters, B.R. Resolution enhancement techniques in microscopy. Eur. Phys. J. H 2013, 38, 281-344. [CrossRef] 
4. Markaki, Y.; Smeets, D.; Fiedler, S.; Schmid, V.J.; Schermelleh, L.; Cremer, T.; Cremer, M. The potential of 3D-FISH and super-resolution structured illumination microscopy for studies of 3D nuclear architecture. Bioessays 2012, 34, 412-426. [CrossRef] [PubMed]

5. Abaza, H.M.; Youssef, S.R.; Saad, A.A.; Kamal, G.M.; Hegazy, M.G.; Ibrahim, R.I.; Annaka, L.M. Detection of 14q32 rearrangements in multiple myeloma, using simultaneous FISH analysis combined with immunofluorescence. Hematol. Oncol. Stem Cell Ther. 2015, 8, 56-63. [CrossRef] [PubMed]

6. Mongelard, F.; Vourch, C.; Robert-Nicoud, M.; Usson, Y. Quantitative assessment of the alteration of chromatin during the course of FISH procedures. Cytometry 1999, 36, 96-101. [CrossRef]

7. Winkler, R.; Perner, B.; Rapp, A.; Durm, M.; Cremer, C.; Greulich, K.O.; Hausmann, M. Labelling quality and chromosome morphology after low temperature FISH analysed by scanning far-field and near-field optical microscopy. J. Microsc. 2003, 209, 23-33. [CrossRef] [PubMed]

8. Zhang, W.I.; Röhse, H.; Rizzoli, S.O.; Opazo, F. Fluorescent in situ hybridization of synaptic proteins imaged with super-resolution STED microscopy. Microsc. Res. Tech. 2014, 77, 517-527. [CrossRef] [PubMed]

9. Raj, A.; van den Bogaard, P.; Rifkin, S.A.; van Oudenaarden, A.; Tyagi, S. Imaging individual mRNA molecules using multiple singly labeled probes. Nat. Methods 2008, 5, 877. [CrossRef] [PubMed]

10. Bachmann, M.; Fiederling, F.; Bastmeyer, M. Practical limitations of superresolution imaging due to conventional sample preparation revealed by a direct comparison of CLSM, SIM and dSTORM. J. Microsc. 2015, 262, 306-315. [CrossRef] [PubMed]

11. Fornasiero, E.F.; Opazo, F. Super-resolution imaging for cell biologists. Bioessays 2015, 37, 436-451. [CrossRef] [PubMed]

12. Anguiano, A. Fluorescence in situ hybridization (FISH): Overview and medical applications. J. Clin. Ligand Assay 2000, 23, 33-42.

13. Wolf, D.; Rauch, J.; Hausmann, M.; Cremer, C. Comparison of the thermal denaturation behaviour of DNA-solutions and metaphase chromosome preparations in suspension. Biophys. Chem. 1999, 81, 207-221. [CrossRef]

14. Rauch, J.; Wolf, D.; Hausmann, M.; Cremer, C. The influence of formamide on thermal denaturation profiles of DNA and metaphase chromosomes in suspension. Z. Naturforsch. C 2000, 55, 737-746. [CrossRef] [PubMed]

15. Hausmann, M.; Winkler, R.; Hildenbrand, G.; Finsterle, J.; Weisel, A.; Rapp, A.; Schmitt, E.; Janz, S.; Cremer, C. COMBO-FISH: Specific labeling of nondenatured chromatin targets by computer-selected DNA oligonucleotide probe combinations. Biotechniques 2003, 35, 564-577. [PubMed]

16. Stuhlmüller, M.; Hausmann, M. Selection of COMBO-FISH probes for multi-purpose applications. J. Theor. Comput. Sci. 2015, 2, 131-132. [CrossRef]

17. Schmitt, E.; Schwarz-Finsterle, J.; Stein, S.; Boxler, C.; Müller, P.; Mokhir, A.; Krämer, R.; Cremer, C.; Hausmann, M. COMBinatorial Oligo FISH: Directed labeling of specific genome domains in differentially fixed cell material and live cells. In Fluorescence In Situ Hybridization (FISH), Protocols and Applications; Bridger, J.M., Volpi, E.V., Eds.; Humana Press: New York, NY, USA, 2010; pp. 185-202.

18. Schmitt, E.; Wagner, J.; Hausmann, M. Combinatorial selection of short triplex forming oligonucleotides for fluorescence in situ hybridisation COMBO-FISH. J. Comput. Sci. 2012, 3, 328-334. [CrossRef]

19. Lemmer, P.; Gunkel, M.; Baddeley, D.; Kaufmann, R.; Urich, A.; Weiland, Y.; Reymann, J.; Müller, P.; Hausmann, M.; Cremer, C. SPDM: Light microscopy with single-molecule resolution at the nanoscale. Appl. Phys. B 2008, 93, 1-12. [CrossRef]

20. Cremer, C.; Kaufmann, R.; Gunkel, M.; Pres, S.; Weiland, Y.; Müller, P.; Ruckelshausen, T.; Lemmer, P.; Geiger, F.; Gegenhard, S.; et al. Superresolution imaging of biological nanostructures by spectral precision distance microscopy. Biotechnol. J. 2011, 6, 1037-1051. [CrossRef] [PubMed]

21. Muller, P.; Schmitt, E.; Jacob, A.; Hoheisel, J.; Kaufmann, R.; Cremer, C.; Hausmann, M. COMBO-FISH enables high precision localization microscopy as a prerequisite for nanostructure analysis of genome loci. Int. J. Mol. Sci. 2010, 11, 4094-4105. [CrossRef] [PubMed]

22. Stuhlmüller, M.; Schwarz-Finsterle, J.; Fey, E.; Lux, J.; Bach, M.; Cremer, C.; Hinderhofer, K.; Hausmann, M.; Hildenbrand, G. In situ optical sequencing and structure analysis of a trinucleotide repeat genome region by localization microscopy after specific COMBO-FISH nano-probing. Nanoscale 2015, 7, 17938-17946. [CrossRef] [PubMed] 
23. Müller, P.; Rößler, J.; Schwarz-Finsterle, J.; Schmitt, E.; Hausmann, M. PNA-COMBO-FISH: From combinatorial probe design in silico to vitality compatible, specific labelling of gene targets in cell nuclei. Exp. Cell Res. 2016, 345, 51-59. [CrossRef] [PubMed]

24. Deininger, P. ALU elements: Know the SINEs. Genome Biol. 2011, 12, 1. [CrossRef] [PubMed]

25. Batzer, M.A.; Deininger, P.L. ALU repeats and human genomic diversity. Nat. Rev. Genet. 2002, 3, 370-379. [CrossRef] [PubMed]

26. Chen, C.; Ara, T.; Gautheret, D. Using ALU elements as polyadenylation sites: A case of retroposon exaptation. Mol. Biol. Evol. 2009, 26, 327-334. [CrossRef] [PubMed]

27. Morales, M.E.; White, T.B.; Streva, V.A.; DeFreece, C.B.; Hedges, D.J.; Deininger, P.L. The contribution of ALU elements to mutagenic DNA double-strand break repair. PLoS Genet. 2015, 11, e1005016. [CrossRef] [PubMed]

28. Lieberman-Aiden, E.; van Berkum, N.L.; Williams, L.; Imakaev, M.; Ragoczy, T.; Telling, A.; Amit, I.; Lajoie, B.R.; Sabo, P.J.; Dorschner, M.O.; et al. Comprehensive mapping of long-range interactions reveals folding principles of the human genome. Science 2009, 326, 289-293. [CrossRef] [PubMed]

29. Gu, Z.; Jin, K.; Crabbe, M.J.C.; Zhang, Y.; Liu, X.; Huang, Y.; Hua, M.; Nan, P.; Zhang, Z.; Zhong, Y. Enrichment analysis of ALU elements with different spatial chromatin proximity in the human genome. Protein Cell 2016, 7, 250-266. [CrossRef] [PubMed]

30. Tsirigos, A.; Rigoutsos, I. ALU and b1 repeats have been selectively retained in the upstream and intronic regions of genes of specific functional classes. PLOS Comput. Biol. 2009, 5, e1000610. [CrossRef] [PubMed]

31. Welsch, P.L.; King, M.C. BRCA1 and BRCA2 and the genetics of breast and ovarian cancer. Hum. Mol. Genet. 2001, 10, 705-713.

32. Chénais, B.; Caruso, A.; Hiard, S.; Casse, N. The impact of transposable elements on eukaryotic genomes: From genome size increase to genetic adaptation to stressful environments. Gene 2012, 509, 7-15. [CrossRef] [PubMed]

33. Wang, C.; Huang, S. Nuclear function of ALUs. Nucleus 2014, 5, 131-137. [CrossRef] [PubMed]

34. Betzig, E.; Patterson, G.H.; Sougrat, R.; Lindwasser, O.W.; Olenych, S.; Bonifacino, J.; Davidson, M.W.; Lippincott-Schwartz, J.; Hess, H.F. Imaging intracellular fluorescent proteins at nanometer resolution. Science 2006, 313, 1642-1645. [CrossRef] [PubMed]

35. Hess, S.T.; Girirajan, T.P.; Mason, M.D. Ultra-high resolution imaging by fluorescence photoactivation localization microscopy. Biophys. J. 2006, 91, 4258-4272. [CrossRef] [PubMed]

36. Huang, B.; Bates, M.; Zhuang, X. Super-resolution fluorescence microscopy. Annu. Rev. Biochem. 2009, 78, 993-1016. [CrossRef] [PubMed]

37. Rust, M.J.; Bates, M.; Zhuang, X. Sub-diffraction-limit imaging by stochastic optical reconstruction microscopy (STORM). Nat. Methods 2006, 3, 793-796. [CrossRef] [PubMed]

38. Heilemann, M.; van de Linde, S.; Schüttpelz, M.; Kaspar, R.; Seefeldt, B.; Mukherjee, A.; Tinnefeld, P.; Sauer, M. Subdiffraction-resolution fluorescence imaging with conventional fluorescent probes. Angew. Chem. Int. Ed. 2008, 47, 6172-6176. [CrossRef] [PubMed]

39. Fölling, J.; Bossi, M.; Bock, H.; Medda, R.; Wurm, C.A.; Hein, B.; Jakobs, S.; Eggeling, C.; Hell, S.W. Fluorescence nanoscopy by ground-state depletion and single-molecule return. Nat. Methods 2008, 5, 943-945. [CrossRef] [PubMed]

40. Dertinger, T.; Colyer, R.; Iyer, G.; Weiss, S.; Enderlein, J. Fast, background-free, 3D super-resolution optical fluctuation imaging (SOFI). Proc. Natl. Acad. Sci. USA 2009, 106, 22287-22292. [CrossRef] [PubMed]

41. Baddeley, D.; Crossman, D.; Rossberger, S.; Cheyne, J.E.; Montgomery, J.M.; Jayasinghe, I.D.; Cremer, C.; Cannell, M.B.; Soeller, C. 4D super-resolution microscopy with conventional fluorophores and single wavelength excitation in optically thick cells and tissues. PLoS ONE 2011, 6, e20645. [CrossRef] [PubMed]

42. Kaufmann, R.; Lemmer, P.; Gunkel, M.; Weiland, Y.; Müller, P.; Hausmann, M.; Baddeley, D.; Amberger, R.; Cremer, C. SPDM: Single Molecule Superresolution of Cellular Nanostructures. In Single Molecule Spectroscopy and Imaging II, Proceedings of SPIE; SPIE BiOS: Biomedical Optics; International Society for Optics and Photonics: San Jose, CA, USA, 2009.

43. Grull, F.; Kirchgessner, M.; Kaufmann, R.; Hausmann, M.; Kebschull, U. Accelerating Image Analysis for Localization Microscopy with FPGAs. In Proceedings of the 21st International Conference on Field Programmable Logic and Applications (FPL), Chania, Greece, 5-7 September 2011. 
44. Lemmer, P.; Gunkel, M.; Weiland, Y.; Müller, P.; Baddeley, D.; Kaufmann, R.; Urich, A.; Eipel, H.; Amberger, R.; Hausmann, M.; et al. Using conventional fluorescent markers for far-field fluorescence localization nanoscopy allows resolution in the 10-nm range. J. Microsc. 2009, 235, 163-171. [CrossRef] [PubMed]

45. Zhang, Y.; Máté, G.; Müller, P.; Hillebrandt, S.; Krufczik, M.; Bach, M.; Kaufmann, R.; Hausmann, M.; Heermann, D.W. Radiation induced chromatin conformation changes analysed by fluorescent localization microscopy, statistical physics, and graph theory. PLoS ONE 2015, 10, e0128555. [CrossRef] [PubMed]

46. Boyd, P.S.; Struve, N.; Bach, M.; Eberle, J.P.; Gote, M.; Schock, F.; Cremer, C.; Kriegs, M.; Hausmann, M. Clustered localization of EGFRvIII in glioblastoma cells as detected by high precision localization microscopy. Nanoscale 2016, 8, 20037-20047. [CrossRef] [PubMed]

47. Kaufmann, R.; Müller, P.; Hildenbrand, G.; Hausmann, M.; Cremer, C. Analysis of Her2/neu membrane protein clusters in different types of breast cancer cells using localization microscopy. J. Microsc. 2011, 242, 46-54. [CrossRef] [PubMed]

48. Becker, J.S.; Nicetto, D.; Zaret, K.S. H3K9me3-dependent heterochromatin: Barrier to cell fate changes. Trends Genet. 2016, 32, 29-41. [CrossRef] [PubMed]

49. Bolzer, A.; Kreth, G.; Solovei, I.; Koehler, D.; Saracoglu, K.; Fauth, C.; Muller, S.; Eils, R.; Cremer, C.; Speicher, M.R.; et al. Three-dimensional maps of all chromosomes in human male fibroblast nuclei and prometaphase rosettes. PLoS Biol. 2005, 3, e157. [CrossRef] [PubMed]

50. RepeatMasker. Available online: http:/ /www.repeatmasker.org (accessed on 7 August 2015).

51. Su, M.; Han, D.; Boyd-Kirkup, J.; Yu, X.; Han, J.J. Evolution of ALU elements toward enhancers. Cell Rep. 2014, 7, 376-385. [CrossRef] [PubMed]

52. Peng, J.C.; Karpen, G.H. Epigenetic regulation of heterochromatic DNA stability. Curr. Opin. Genet. Dev. 2008, 18, 204. [CrossRef] [PubMed]

53. Sander, J.; Ester, M.; Kriegel, H.P.; Xu, X. Density-based clustering in spatial databases: The algorithm gdbscan and its applications. Data Min. Knowl. Discov. 1998, 2, 169-194. [CrossRef]

54. Braden, B. The surveyor's area formula. Coll. Math. J. 1986, 17, 326-337. [CrossRef]

55. Chen, C.; Gentles, A.J.; Jurka, J.; Karlin, S. Genes, pseudogenes, and ALU sequence organization across human chromosomes 21 and 22. Proc. Natl. Acad. Sci. USA 2002, 99, 2930-2935. [CrossRef] [PubMed]

56. Lorat, Y.; Schanz, S.; Schuler, N.; Wennemuth, G.; Rübe, C.; Rübe, C.E. Beyond repair foci: DNA double-strand break repair in euchromatic and heterochromatic compartments analyzed by transmission electron microscopy. PLoS ONE 2012, 7, e38165. [CrossRef] [PubMed]

57. Schwarz-Finsterle, J.; Stein, S.; Großmann, C.; Schmitt, E.; Trakhtenbrot, L.; Rechavi, G.; Amariglio, N.; Cremer, C.; Hausmann, M. Comparison of triple helical COMBO-FISH and standard FISH by means of quantitative microscopic image analysis of abl/bcr positions in cell nuclei. J. Biochem. Biophys. Methods 2007, 70, 397-406. [CrossRef] [PubMed]

58. Weiland, Y.; Lemmer, P.; Cremer, C. Combining FISH with localisation microscopy: Super-resolution imaging of nuclear genome nanostructures. Chromosome Res. 2011, 19, 5-23. [CrossRef] [PubMed]

59. Falk, M.; Hausmann, M.; Lukášová, E.; Biswas, A.; Hildenbrand, G.; Davídková, M.; Krasavin, E.; Kleibl, Z.; Falkova, I.; Jezkova, L.; et al. Giving OMICS spatiotemporal dimensions by challenging microscopy: From functional networks to structural organization of cell nuclei elucidating mechanisms of complex radiation damage response and chromatin repair-PART A (Radiomics). Crit. Rev. Eukaryot. Gene Expr. 2014, 24, 205-223. [CrossRef] [PubMed]

60. Falk, M.; Hausmann, M.; Lukášová, E.; Biswas, A.; Hildenbrand, G.; Davídková, M.; Krasavin, E.; Kleibl, Z.; Falkova, I.; Jezkova, L.; et al. Giving OMICS spatiotemporal dimensions by challenging microscopy: From functional networks to structural organization of cell nuclei elucidating mechanisms of complex radiation damage response and chromatin repair-PART B (Structuromics). Crit. Rev. Eukaryot. Gene Expr. 2014, 24, 225-247. [CrossRef] [PubMed]

61. Gutierrez, C.; Schiff, R. HER2: Biology, detection, and clinical implications. Arch. Pathol. Lab. Med. 2011, 135, 55-62. [PubMed]

(C) 2017 by the authors. Licensee MDPI, Basel, Switzerland. This article is an open access article distributed under the terms and conditions of the Creative Commons Attribution (CC BY) license (http:/ / creativecommons.org/licenses/by/4.0/). 